\title{
High glucose increases the formation and pro-oxidative activity of endothelial microparticles
}

\author{
Dylan Burger $^{1,2} \cdot$ Maddison Turner $^{1,2} \cdot$ Fengxia Xiao $^{1} \cdot$ Mercedes N. Munkonda ${ }^{1} \cdot$ \\ Shareef Akbari ${ }^{1,2} \cdot$ Kevin D. Burns ${ }^{1,2,3}$
}

Received: 14 February 2017 / Accepted: 18 May 2017 /Published online: 10 June 2017

(C) Springer-Verlag GmbH Germany 2017

\begin{abstract}
Aims/hypothesis Individuals with diabetes exhibit increases in circulating endothelial microparticles (eMPs, also referred to as endothelial microvesicles), which are associated with endothelial dysfunction and a heightened risk of cardiovascular complications. We have shown that eMPs are markers and mediators of vascular injury although their role in diabetes is unclear. We hypothesised that the composition and biological activity of eMPs are altered in response to high glucose exposure. We assessed the effects of high glucose on eMP formation, composition and signalling in cultured HUVECs. Methods eMPs were isolated from the media of HUVECs cultured under conditions of normal glucose $\left(\mathrm{eMP}^{\mathrm{NG}}\right)$, high glucose $\left(\mathrm{eMP}^{\mathrm{HG}}\right)$ or osmotic control of L-glucose $\left(\mathrm{eMP}^{\mathrm{LG}}\right)$. eMP size, concentration and surface charge were assessed by nanoparticle tracking analysis and flow cytometry. eMP protein composition was assessed by liquid chromatography-tandem mass spectrometry, and eMP-mediated effects on coagu-
\end{abstract}

Dylan Burger and Maddison Turner contributed equally to this study.

Electronic supplementary material The online version of this article (doi:10.1007/s00125-017-4331-2) contains peer-reviewed but unedited supplementary material, which is available to authorised users.

Dylan Burger

dburger@uottawa.ca

$\checkmark$ Kevin D. Burns

kburns@ ottawahospital.on.ca

1 Kidney Research Centre, Ottawa Hospital Research Institute, University of Ottawa, 1967 Riverside Drive, Room 535, Ottawa, ON K1H 7W9, Canada

2 Department of Cellular and Molecular Medicine, University of Ottawa, 2513-451 Smyth Road, Ottawa, ON K1H 8M5, Canada

3 Division of Nephrology, Department of Medicine, The Ottawa Hospital, Ottawa, ON, Canada lation, reactive oxygen species (ROS) production and vessel function were assessed.

Results Exposure of HUVECs to high glucose for $24 \mathrm{~h}$ caused a threefold increase in eMP formation, increased mean particle size $(269 \pm 18 \mathrm{~nm}$ vs $226 \pm 11 \mathrm{~nm})$ and decreased surface charge. Compared with $\mathrm{eMP}^{\mathrm{NG}}$ or $\mathrm{eMP}^{\mathrm{LG}}, \mathrm{eMP}^{\mathrm{HG}}$ possessed approximately threefold greater pro-coagulant activity, stimulated HUVEC ROS production to a greater extent $(\sim 250 \%$ of $\mathrm{eMP}^{\mathrm{NG}}$ ) and were more potent inhibitors of endothelialdependent relaxation. Proteomic analysis of eMPs identified 1212 independent proteins of which 68 were exclusively found in $\mathrm{eMP}^{\mathrm{HG}}$. Gene ontology analysis revealed that $\mathrm{eMP}^{\mathrm{HG}}$-exclusive proteins were associated with signalling pathways related to blood coagulation, cell signalling and immune cell activation.

Conclusions/interpretation Our results indicate that elevated glucose is a potent stimulus for eMP formation that also alters their molecular composition leading to increased bioactivity. Such effects may contribute to progressive endothelial injury and subsequent cardiovascular complications in diabetes.

Keywords Endothelial $\cdot$ Extracellular vesicle ·

Microparticles $\cdot$ Microvesicle $\cdot$ Reactive oxygen species ·

Vascular

$\begin{array}{ll}\text { Abbreviations } \\ \text { ApoE } & \text { Apolipoprotein E } \\ \mathrm{eMP} & \begin{array}{l}\text { Endothelial microparticle } \\ \text { Endothelial microparticles cultured under } \\ \text { eMP }\end{array} \\ & \begin{array}{l}\text { conditions of high glucose } \\ \text { Endothelial microparticles cultured under } \\ \text { eMP }\end{array} \\ & \begin{array}{l}\text { conditions of osmotic control of L-glucose } \\ \text { Endothelial microparticles cultured } \\ \text { eMP }\end{array} \\ & \text { under conditions of normal glucose }\end{array}$




$\begin{array}{ll}\text { HMEC } & \begin{array}{l}\text { Human dermal microvascular } \\ \text { endothelial cell } \\ \text { Liquid chromatography-tandem mass } \\ \text { spectrometry } \\ \text { microRNA }\end{array} \\ \text { miRNA } & \text { Nanoparticle tracking analysis } \\ \text { NTA } & \text { Reactive oxygen species } \\ \text { ROS } & \end{array}$

\section{Introduction}

Cells release a variety of extracellular vesicles including apoptotic bodies, exosomes and membrane-derived microparticles (also referred to as microvesicles) [1-4]. These extracellular vesicles play an increasingly recognised role in intercellular signalling through surface interactions, immune modulation, and horizontal transfer of protein and nucleic acids. Unique among the various extracellular vesicles, microparticles $(100-1000 \mathrm{~nm}$ in size) are formed from small, outward-facing membrane blebs that are released into the extracellular compartment. Notably, microparticles are formed under conditions of cell stress and contain cytosolic and membrane protein, RNA and microRNA (miRNA) [1,2]. Because microparticles are formed at the earliest stages of cell injury and their composition is reflective of their source cells, microparticles are emerging as biomarkers of underlying pathology $[1,2]$.

Among the most widely studied populations of microparticles are endothelial-derived microparticles (eMPs). eMPs are released from both activated and apoptotic endothelial cells and can be found in human plasma samples [5, 6]. Levels of circulating eMPs are increased in human diseases including diabetes mellitus, end-stage kidney disease and hypertension (reviewed in [1]), and such elevations predict adverse cardiovascular events [7, 8]. In addition to their utility as biomarkers, eMPs are also novel mediators of intercellular communication [9]. In this regard, we and others have shown that eMPs induce intracellular signalling responses leading to oxidative stress, inflammation, senescence and apoptosis [10-14].

The composition of eMPs and the potency of their effects on intracellular signalling responses are modulated by several factors. For example, eMPs formed during endothelial cell activation are enriched in surface membrane CD51 and CD54 in comparison with eMPs formed during apoptosis [6]. Similarly, using a proteomic-based approach, Peterson et al identified marked changes in composition of eMPs derived from cells exposed to TNF $\alpha$ vs plasminogen activator inhibitor type 1 [15]. In vivo, eMPs isolated from individuals with the metabolic syndrome induce vascular dysfunction when injected into mice, while those from healthy participants have no effect [16]. Jansen et al reported that eMPs formed under high glucose conditions increase atherosclerotic lesions in apolipoprotein $\mathrm{E}(\mathrm{ApoE})^{-/-}$mice: an effect not seen from eMPs formed under normal glucose conditions [17].
Moreover, eMP miRNA profiles are altered in type 2 diabetes with reductions in miR-26a (anti-apoptotic in endothelial cells) and miR-126 (pro-angiogenic) [18]. Collectively these studies suggest that the diabetic microenvironment may influence the composition and bioactivity of eMPs; however, the precise changes to eMPs in response to high glucose are not well understood.

In the present study, we assessed the impact of high glucose on the formation, size, protein composition and bioactivity of eMPs. We hypothesised that high glucose would alter the composition of eMPs, contributing to increased adverse effects on endothelial cell function.

\section{Methods}

More detailed methods can be found in the electronic supplementary material (ESM) Methods.

Culture of endothelial cells HUVECs and human dermal microvascular endothelial cells (HMECs) were obtained from the American Type Culture Collection (ATCC, Manassas, VA, USA) and cultured as described previously with modification [19]. Cells were seeded on attachment factor (ThermoFisher Scientific, Mississauga, ON, Canada) coated dishes and grown in a $\mathrm{CO}_{2}$ incubator $(5 \%)$ at $37^{\circ} \mathrm{C}$. Culture medium consisted of Endomax medium with EMS-18 (Wisent Bioproducts, St Bruno, QC, Canada), $100 \mathrm{U} / \mathrm{ml}$ penicillin/streptomycin (Wisent Bioproducts) and 10\% FBS (ATCC). Cells were verified to be mycoplasma free using the MycoAlert Mycoplasma Detection Kit (Lonza, Basel, Switzerland).

eMP generation and isolation eMPs were isolated from the medium of cultured HUVECs treated with normal glucose (5.6 mmol/1; eMP ${ }^{\mathrm{NG}}$ ), high glucose (normal glucose $+19.4 \mathrm{mmol} / \mathrm{l}$ D-glucose [ThermoFisher Scientific]; $\mathrm{eMP}^{\mathrm{HG}}$ ) or an osmotic control (normal glucose $+19.4 \mathrm{mmol} / \mathrm{l} \mathrm{L}$-glucose [Sigma Aldrich, Oakville, ON, Canada]; $\mathrm{eMP}^{\mathrm{LG}}$ ). Media were collected and eMPs were isolated by differential centrifugation as described previously $[12,13]$.

Nanoparticle tracking analysis Particle size and concentration were assessed by nanoparticle tracking analysis (NTA) using the ZetaView PMX 110 Multiple Parameter Particle Tracking Analyzer (Particle Metrix, Meerbusch, Germany) in size mode as described previously [20].

Detection of microparticles by flow cytometry Isolated microparticles were quantified using a MoFlo Fluorescence Activated Cell Sorter with Summit software (Dako Canada, Mississauga, ON, Canada) as described previously [12]. 
Measurement of zeta potential Zeta potential is a measure of particle surface charge, dependent on protein/phospholipid composition and particle stability. Zeta potential was determined from the electrophoretic mobility of the vesicles in solution using the ZetaView PMX 110 Multiple Parameter Particle Tracking Analyzer (Particle Metrix) in zeta potential mode using ZetaView software (version 8.02.28). Samples were suspended in dilute PBS (1:20 PBS, double-distilled $\mathrm{H}_{2} \mathrm{O}$ to adjust conductivity to approximately $500 \mu \mathrm{S} / \mathrm{cm}$ ) and zeta potential readings were obtained at $21^{\circ} \mathrm{C}$.

eMP pro-coagulant activity eMP pro-coagulant activity was measured using the Zymuphen MP-Activity Kit (Aniara, West Chester, OH, USA) as described, with modifications [21]. Pilot experiments were conducted to optimise the working range of the assay. eMP isolates (100 ng) were loaded into wells of a 96-well plate. Thrombin generation was quantified with a proprietary thrombin-specific chromogenic substrate and used to quantify the pro-coagulant activity. Absorbance was read on the ELx808 Absorbance microplate reader (Biotek Instruments, Winooski, VT, USA) and eMP coagulant activity was expressed as a relative absorbance value (measure at $\lambda=405 \mathrm{~nm})$.

Phosphatidylserine content Levels of phosphatidylserine in eMPs were measured using a human phosphatidylserine ELISA kit (Elabscience, Beijing, People's Republic of China) according to manufacturer's instructions. Absorbance was read $(\lambda=450 \mathrm{~nm})$ on the ELx808 Absorbance microplate reader (Biotek Instruments) and expressed as phosphatidylserine equivalents (ng/ml).

Reactive oxygen species production HUVEC superoxide $\left(\mathrm{O}_{2}{ }^{-}\right)$production following eMP or PBS treatment was assessed by HPLC using the fluorescent probe dihydroethidium (Invitrogen, Burlington, ON, Canada) as described $[12,13,19]$. Cells were exposed to $10 \mu \mathrm{g} / \mathrm{ml}$ of eMPs for $4 \mathrm{~h}$, a dose we previously identified to induce intracellular responses in cultured mouse aortic endothelial cells [11-13].

Intracellular reactive oxygen species (ROS) formation was measured using 7'-dichlorofluorescein diacetate (DCF-DA) (ThermoFisher Scientific) as described [22]. Briefly, cells were incubated with $5 \mu \mathrm{mol} / \mathrm{l} \mathrm{DCF}-\mathrm{DA}$ for $30 \mathrm{~min}$ following eMP and PBS treatments $(10 \mu \mathrm{g} / \mathrm{ml}, 4 \mathrm{~h}$, determined as above), and fluorescence was measured on a FLUOstar Galaxy microplate reader (BMG Lab Technologies, Ortenberg, Germany) (excitation $485 \mathrm{~nm}$, emission $520 \mathrm{~nm}$ ).

Myography Mesenteric arteries were isolated from male C57BL/6 mice (age 3-4 months, 20-30 g; The Jackson Laboratory, Bar Harbour, ME, USA) following euthanasia with $\mathrm{CO}_{2}$ asphyxiation. Prior to euthanasia, mice were housed in a ventilated rack system in standard shoebox cages with corncob bedding (Harlan Teklad, Indianapolis, IN, USA). Mice were provided food and water ad libitum and housed on a $12 \mathrm{~h}$ day-night cycle (lights on at 07:00 h); room temperature was $22 \pm 1^{\circ} \mathrm{C}$ and humidity was $50 \pm 5 \%$. The study was approved by the Animal Ethics Committee of the University of Ottawa and performed according to the recommendations of the Canadian Council for Animal Care.

Endothelium-dependent relaxation was measured in the presence or absence of eMP populations $(1 \mu \mathrm{g} / \mathrm{ml})$ as described [23]. Vessels were randomly assigned to treatment groups, incubated with $\mathrm{eMP}^{\mathrm{NG}}$ and $\operatorname{eMP}^{\mathrm{HG}}(1 \mu \mathrm{g} / \mathrm{ml})$, or equivalent volume of PBS, for $30 \mathrm{~min}$ and subsequently preconstricted with phenylephrine, as above. Effects of eMPs on endothelium-dependent relaxation were then assessed by measuring dilatory responses to acetylcholine ( $1 \mathrm{nmol} / \mathrm{l} ; 10 \mu \mathrm{mol} / \mathrm{l})$. Analysis was performed, in a blinded fashion, using LabChart 7 Pro software (ADInstruments, Colorado Springs, CO, USA).

Proteomic and bioinformatics analysis eMPs were isolated from the medium of cultured HUVECs as described above. Equivalent eMP protein extracts from normal glucose, high glucose and osmotic-controlled treatments were separated on a 4-15\% Mini-PROTEAN TGX Gel (Bio-Rad Laboratories, Hercules, CA, USA) ( $n=3$ per eMP treatment condition), bands were excised using a gel excision tool (The Gel Company, San Francisco, CA, USA) and digested in trypsin (Promega, Madison, WI, USA) [24]. Samples were purified by ZipTip (Millipore, Etobicoke, ON, Canada), concentrated by (Eppendorf Vacufuge; ThermoFisher Scientific) and re-suspended in $0.1 \%$ formic acid.

Peptides were analysed by liquid chromatography-tandem mass spectrometry (LC-MS/MS). Nanoflow HPLC solvents contained $0.1 \%$ formic acid and 5\% DMSO [25]. MS scans were acquired in Fourier transform MS mode at a resolution setting of 60,000. MS/MS scans were acquired in ion trap collision-induced dissociation mode using data-dependent acquisition of the top five ions from each MS scan.

MASCOT software, version 2.5.1 (Matrix Science, Boston, MA, USA), was used to infer peptide and protein identities from the mass spectra. Identified MASCOT peptides and proteins were validated with Scaffold (version Scaffold 4.7.3; Proteome Software, Portland, OR, USA). Proteins with at least two spectral counts in all three LC-MS/MS samples ( $n=3$ per eMP treatment condition) were included in the ClueGO functional analysis [26, 27]. Cytoscape (3.4.0, Java version: 1.8.0 121) was used with the ClueGO and CluePedia plug-ins (v2.3.3 and v1.3.3) [27]. Proteins exclusive to the $\mathrm{eMP}^{\mathrm{HG}}$ population were analysed for functional enrichment of gene ontology biological processes (updated 23 February 2017) and Reactome pathways (updated 1 March 2017). 
Statistical analysis Results are expressed as mean \pm SEM and were analysed using the Mann-Whitney $U$ test or KruskalWallis test with Dunn's post hoc test using Prism 5.0 software (GraphPad Software, La Jolla, CA, USA). A $p$ value $<0.05$ was considered significant.

\section{Results}

High glucose induces eMP formation in vitro Increases in circulating eMP levels are associated with elevated $\mathrm{HbA}_{1 \mathrm{c}}$ in diabetic individuals, suggesting a role for glucose in eMP formation [28]. We examined the effect of high glucose on eMP release from cultured HUVECs using flow cytometry and NTA. As shown in Fig. 1, HUVECs cultured under high glucose conditions exhibited significant increases in eMP release compared with HUVECs cultured under normal glucose or osmotic-controlled conditions measured by flow cytometry (Fig. 1a) and NTA (Fig. 1b). A similar increase in microparticle formation was seen in HMECs exposed to high glucose $\left(1.4 \pm 0.1 \times 10^{7}\right.$ microparticles $/ \mu \mathrm{g}$ vs normal glucose $1.0 \pm 0.3$ microparticles $/ \mu \mathrm{g}, p<0.05)$, while L-glucose controls were not altered $(0.9 \pm 0.4$ microparticles $/ \mu \mathrm{g}, p=\mathrm{NS})$.

High glucose alters the mean size and surface charge of eMPs We next assessed whether high glucose altered the physical characteristics of eMPs by NTA and zeta potential. The mean size of $\mathrm{eMP}^{\mathrm{HG}}$ was significantly larger than that of $\mathrm{eMP}^{\mathrm{NG}}$ and $\mathrm{eMP}^{\mathrm{LG}}$ (Fig. 2a-d). Similarly, eMP ${ }^{\mathrm{HG}}$ from HMECs were significantly larger than $\mathrm{eMP}^{\mathrm{NG}}(187 \pm 14 \mathrm{~nm}$ vs normal glucose $148 \pm 3, p<0.05$ ), while $\mathrm{eMP}^{\mathrm{LG}}$ were not $(150 \pm 3 \mathrm{~nm}, p=\mathrm{NS})$. $\mathrm{eMP}^{\mathrm{HG}}$ had a greater negative surface charge compared with eMP ${ }^{\mathrm{NG}}$ (Fig. 2e). The surface charge of $\mathrm{eMP}^{\mathrm{LG}}$ was not significantly different from that of $\mathrm{eMP}^{\mathrm{NG}}$ (not shown).

High glucose increases the pro-oxidative and procoagulant effects of eMPs released from HUVECs Pro-coagulant capacity is increased in eMPs from diabetic individuals and correlates with $\mathrm{HbA}_{1 \mathrm{c}}$ levels [29]. We therefore

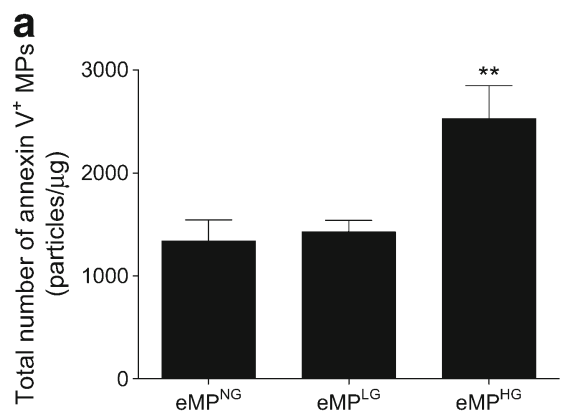

Fig. 1 Effects of high glucose on eMP formation in cultured HUVECs. Vesicle levels were assessed by (a) annexin V labelling and flow cytometry or (b) NTA. Data have been normalised to total cell protein and are assessed the effect of high glucose on the pro-coagulant and pro-oxidative effects of eMPs. eMP ${ }^{\mathrm{HG}}$ had increased procoagulant activity compared with $\mathrm{eMP}^{\mathrm{NG}}$ and $\mathrm{eMP} \mathrm{P}^{\mathrm{LG}}$ $\left(p<0.001 \mathrm{vs} \mathrm{eMP}^{\mathrm{NG}}\right.$ and $\mathrm{eMP}^{\mathrm{LG}}, n=4$, Fig. 3a). eMP procoagulant activity has been partially attributed to surface phosphatidylserine $[30,31]$. As shown in Fig. $3 b$, eMP ${ }^{\mathrm{HG}}$ had an approximately threefold increase in phosphatidylserine content compared with eMP ${ }^{\mathrm{NG}}$ and $\mathrm{eMP}^{\mathrm{LG}}$.

Intracellular HUVEC ROS production was increased following $\mathrm{eMP}^{\mathrm{NG}}$ and $\mathrm{eMP}^{\mathrm{LG}}$ compared with PBS-treated controls ( $p<0.001$ vs untreated controls, $n=3$, Fig. $3 \mathrm{c}$ ). ROS production was further increased with $\mathrm{eMP}^{\mathrm{HG}}$ treatment $(p<0.001$, Fig. $3 \mathrm{c})$. In addition to intracellular ROS production, HUVEC $\mathrm{O}_{2}^{-}$production was increased following $\mathrm{eMP}^{\mathrm{HG}}$ treatment $\left(p<0.05 \mathrm{vs} \mathrm{eMP}^{\mathrm{NG}}\right.$ and $\mathrm{eMP}^{\mathrm{LG}}$, Fig. 3d).

High glucose increases the capacity of eMPs to impair endothelial-dependent vasorelaxation To determine the effect of eMPs on endothelium-dependent vasorelaxation, we assessed acetylcholine-mediated relaxation in phenylephrinepreconstricted arteries exposed to $\mathrm{eMP}^{\mathrm{NG}}$ and $\mathrm{eMP}^{\mathrm{HG}}$ (Fig. 4). Arteries exposed to $\mathrm{eMP}^{\mathrm{NG}}$ in the vessel bath displayed a rightward shift in their relaxation curve and a significant reduction in maximal relaxation compared with PBS-treated arteries ( $p<0.05$ vs controls). Arteries treated with $\mathrm{eMP}^{\mathrm{HG}}$ displayed a further rightward shift with a significant reduction in both maximal relaxation and sensitivity to acetylcholine, suggesting a more potent impairment in vascular reactivity.

High glucose alters the protein composition of eMPs released from HUVECs To gain insight into changes in protein composition that may contribute to altered eMP activity we conducted proteomic analysis of high-glucose-treated eMP isolates. LC-MS/MS analysis identified 1212 independent proteins with a minimum of two spectral counts per sample. Of the 1212 proteins identified, 724 were common to all treatment groups, while 68 were unique to the $\mathrm{eMP}^{\mathrm{HG}}$ population and 111 were unique to the $\mathrm{eMP}^{\mathrm{LG}}$ population (Fig. 5a, ESM Table 1).

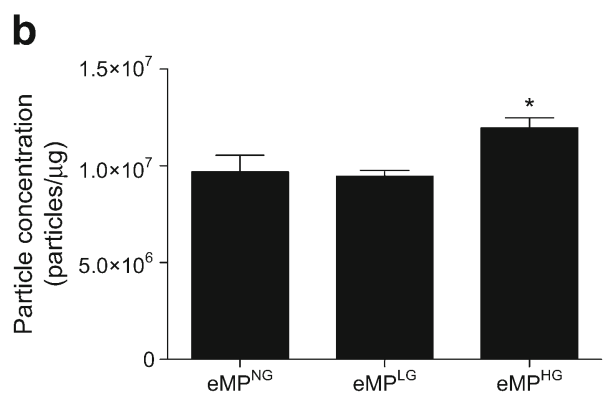

presented as means \pm SEM. $* p<0.05$ and $* * p<0.01 \mathrm{vs} \mathrm{eMP}^{\mathrm{NG}}$ and $\mathrm{eMP}^{\mathrm{LG}}$, respectively $(n=5)$. MPs, microparticles 

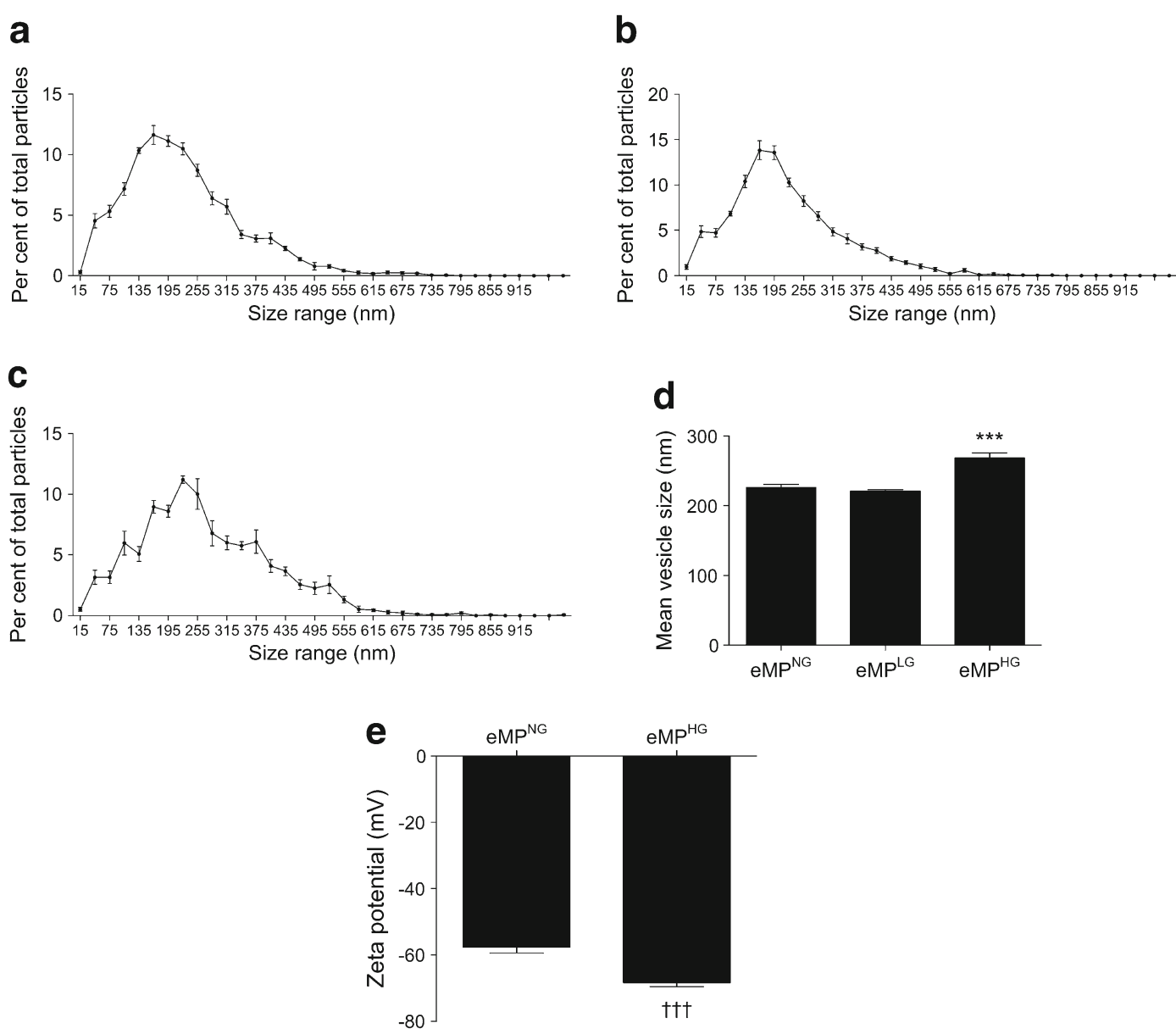

Fig. 2 High glucose increases the size and surface charge of eMPs released by cultured HUVECs. Distribution of the size of eMP particles: (a) $\mathrm{eMP}^{\mathrm{NG}},(\mathbf{b}) \mathrm{eMP}^{\mathrm{LG}}$, (c) $\mathrm{eMP}^{\mathrm{HG}}$. (d) Mean particle size $(\mathrm{nm})$ of each vesicle population: $\mathrm{eMP}^{\mathrm{NG}} 226.15 \pm 10.60 \mathrm{~nm}(n=5)$, eMP $\mathrm{eM}^{\mathrm{LG}}$

To examine the functional nature of protein alterations in $\mathrm{eMP}^{\mathrm{HG}}$, we conducted a functional analysis for enriched biological processes and downstream pathways using ClueGO (Fig. 5b). Notably, protein localisation to the membrane was an over-represented pathway, while nuclear processes were not enriched, which is consistent with microparticle isolation with little contamination from larger, apoptotic bodies (Fig. 5b). Gene ontology biological processes and Reactome pathways associated with $\mathrm{eMP}^{\mathrm{HG}}$-exclusive proteins included those involved in haemostasis, hexose metabolism, cell surface interactions at the vascular wall and oxidation-reduction processes (Fig. 5b, ESM Table 2).

\section{Discussion}

The purpose of the present study was to assess the impact of high glucose on the formation, biological activity and composition of eMPs. Novel findings include: (1) high glucose exposure increases eMP release from cultured HUVECs; (2) high glucose alters the make-up of eMPs, with an increase in

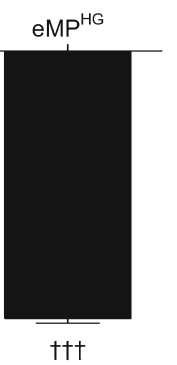

$221.18 \pm 4.80 \mathrm{~nm}(n=6)$ and $\mathrm{eMP}^{\mathrm{HG}} 268.55 \pm 18.02 \mathrm{~nm}(n=6)$; *** $p<0.001 \mathrm{vs} \mathrm{eMP}{ }^{\mathrm{NG}}$ and $\mathrm{eMP}^{\mathrm{LG}}$. (e) Zeta potential was calculated from electrophoretic mobilities; ${ }^{\dagger \dagger} p<0.001$ vs eMP ${ }^{\mathrm{NG}}(n=7)$. Data are presented as mean $\pm \mathrm{SEM}$

size, changes in surface charge and enrichment in phosphatidylserine; (3) eMPs formed in high glucose exhibit increased pro-coagulant activity, are more potent inducers of ROS production and exert greater impairment of endotheliumdependent vasorelaxation compared with eMPs formed in normal glucose; and (4) eMPs formed in high glucose possess a distinct molecular composition with proteins associated with hexose metabolism, haemostasis, cell surface interactions at the vascular wall, and oxidation-reduction processes. Together, these results suggest a central role for high glucose in increasing both formation and bioactivity of eMPs which could, in turn, contribute to greater vascular dysfunction in diabetes.

Diabetes induces endothelial dysfunction, an independent predictor of poor prognosis linked to retinal and renal damage, myocardial infarction and stroke. While a number of factors have been implicated in the development of endothelial dysfunction in diabetes, hyperglycaemia is considered to play a key causative role, since intensive glycaemic control reduces endothelial dysfunction and improves prognosis in both type 1 and type 2 diabetes [32-34]. Also associated with endothelial 
a

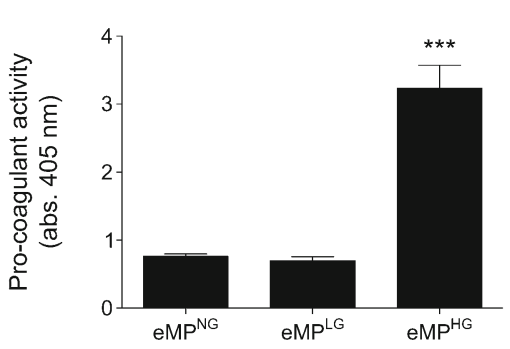

C

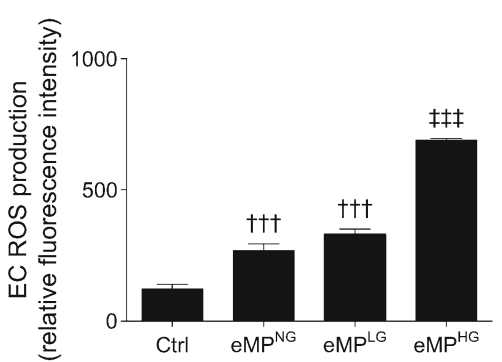

Fig. 3 Effect of high glucose on the pro-coagulant activity, phosphatidylserine content and pro-oxidative activity of eMPs released from cultured HUVECs. (a) Pro-coagulant activity was assessed in equivalent amounts of eMPs using a Zymuphen MP-Activity Kit according to manufacturer's instructions $(n=4)$. (b) Phosphatidylserine content was measured by ELISA $(n=4)$. $(\mathbf{c}, \mathbf{d})$ Cells were treated with respective eMP preparations $(10 \mu \mathrm{g} / \mathrm{ml}, 4 \mathrm{~h})$, and endothelial cell (EC) ROS production

injury/dysfunction is the formation of eMPs. Levels of circulating eMPs are increased in both type 1 and type 2 diabetes $[28,29,35]$. When glycaemia is controlled, these elevated levels are reduced $[36,37]$. These observations implicate hyperglycaemia as a critical determinant of eMP levels in diabetes. In the present study, we observed that $24 \mathrm{~h}$ high glucose exposure results in increased eMP levels from HUVECs. This effect could not be attributed to osmotic stress, as L-glucose was without effect. We studied the acute effect of $25 \mathrm{mmol} / \mathrm{L}$-glucose on endothelial cell health to examine the effects of uncontrolled glucose on vesicle composition. Our

\section{b}

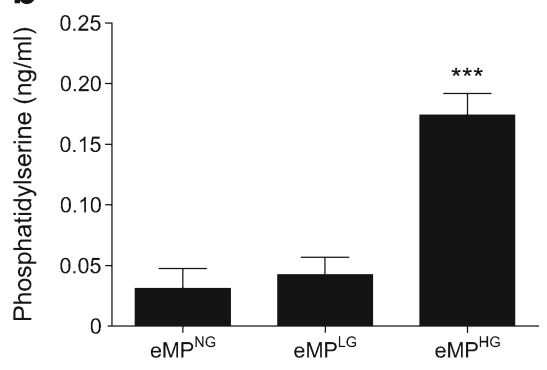

d

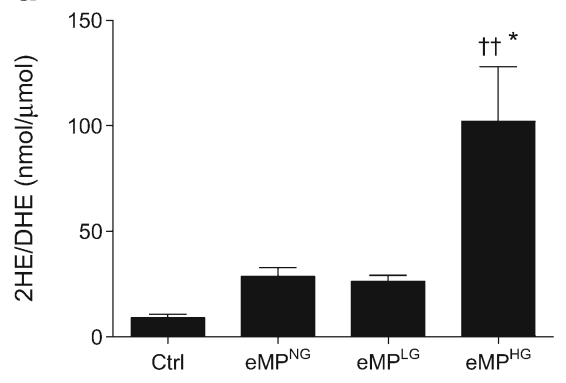

was assessed by dichlorofluorescein fluorescence (c) or dihydroethidium (DHE) HPLC (expressed as the ratio of 2-hydroxyethidium [2-HE]/DHE) (d) $(n=3) . * p<0.05, * * * p<0.001$ vs eMP ${ }^{\mathrm{NG}}$ and eMP ${ }^{\mathrm{LG}} ;{ }^{\dagger \dagger} p<0.01$, ${ }^{\dagger \dagger} p<0.001$ vs untreated controls (Ctrl); ${ }^{\star * t} p<0.001$ vs eMP ${ }^{\mathrm{NG}}$, eMP $\mathrm{eMG}^{\mathrm{LG}}$ and untreated controls. Data are presented as mean \pm SEM. Abs., absorbance

results remain consistent with prior observations of elevated podocyte microparticle formation in response to high glucose [38], and increased microparticle release from mesangial cells exposed to high glucose [39]. Given the variety of cells that release microparticles in response to high glucose it is possible that increased microparticle release represents a ubiquitous response to glucose-induced cell stress. In addition to increased formation, we observed an increase in vesicle size and zeta potential following high glucose exposure. We hypothesise that the increase in size is a result of increased protein packaging into the vesicles and increased surface

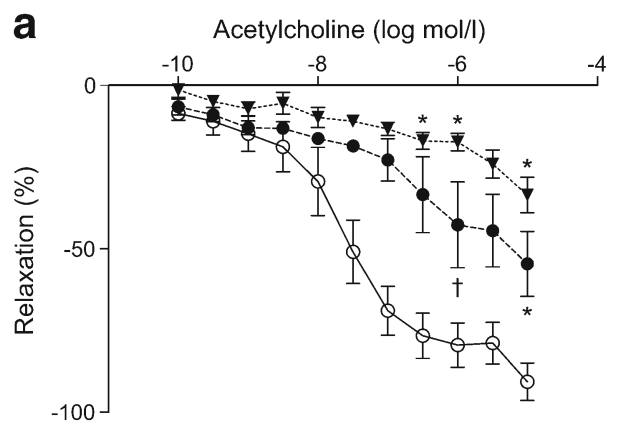

Fig. 4 Effect of high glucose on the vascular effects of eMPs assessed via ex vivo endothelial-dependent vasorelaxation in isolated arteries. (a) Concentration-response curve to acetylcholine in untreated control vessels $\left(n=8\right.$; open circles, solid line) or those incubated with $\operatorname{eMP}^{\mathrm{NG}}(n=6$; black circles, dashed line) or $\mathrm{eMP}^{\mathrm{HG}}(n=5$; black triangles, dashed line)

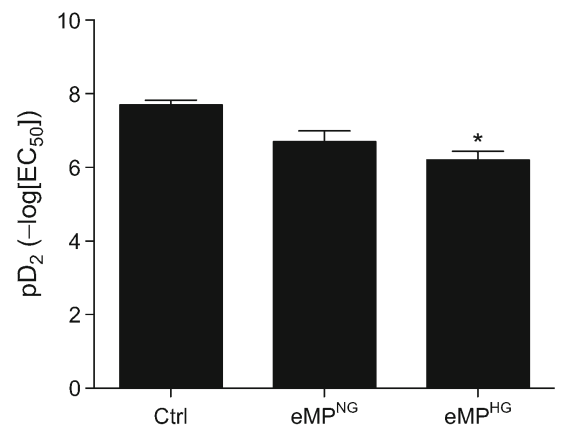

( $1 \mu \mathrm{g} / \mathrm{ml}, 30 \mathrm{~min})$. Acetylcholine data are log-transformed $\left(\log _{10}\right)$. $* p<0.05$ vs control vessels at corresponding dose; ${ }^{\dagger} p<0.05$ vs $\mathrm{eMP}^{\mathrm{HG}}$-treated vessels at corresponding dose. (b) Sensitivity to acetylcholine, expressed as $\mathrm{pD}_{2}\left(-\log _{10}\right.$ of half maximal effective concentration $\left.\left[\mathrm{EC}_{50}\right]\right)$. Data are presented as mean $\pm \mathrm{SEM} ; * p<0.05$ vs controls $(\mathrm{Ctrl})$ 


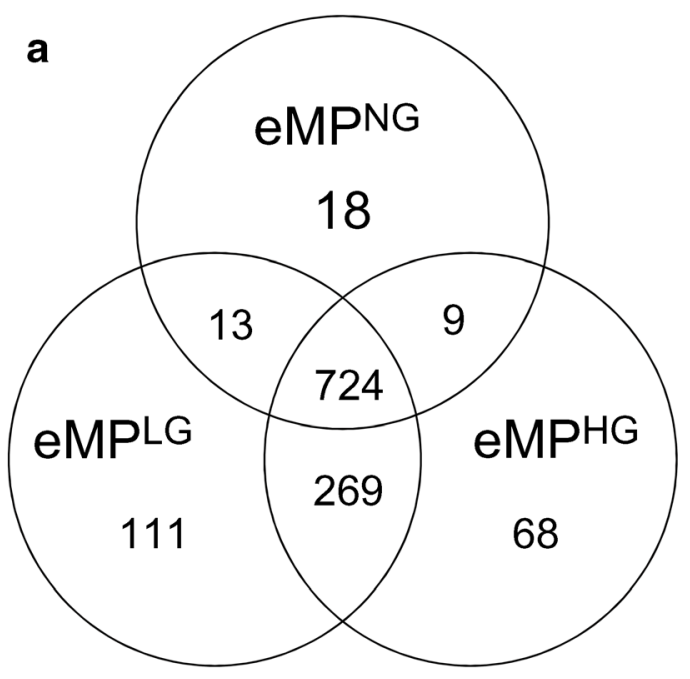

Fig. 5 High glucose changes the proteomic composition of eMPs. (a) Venn diagram depicts differences in protein composition among eMP populations. (b) Functional analysis (gene ontology biological processes

phosphatidylserine content. Thus, we looked at the vesicles' proteomic signature and phosphatidylserine content. Phosphatidylserine is a negatively charged phospholipid, typically present on the inner leaflet of the cell membrane. However, under cellular stress an enzymatic response causes phosphatidylserine to become externalised, which might have caused the increased electronegativity in our $\mathrm{eMP}^{\mathrm{HG}}$ population.

In the present study, we observed an increase in eMP procoagulant capacity following high glucose exposure. Our data provide a possible explanation for the observations of Sabatier and colleagues, who found that the pro-coagulant activity of circulating microparticles correlated with $\mathrm{HbA}_{1 \mathrm{c}}$ in type 1 diabetic individuals [28]. In the present study, $\mathrm{eMP}^{\mathrm{HG}}$ were also enriched in phosphatidylserine (a primary determinant of microparticle pro-coagulant activity $[30,31])$ and were more electronegative. One possibility is that eMPs may be mediators of the coagulation cascade by acting as catalytic surfaces, whereby coagulation factors (Factor $\mathrm{Xa}, \mathrm{V}$ and prothrombin) assemble into a complex and initiate the coagulation cascade on the negatively charged phosphatidylserine-enriched vesicle surface. Nevertheless, we cannot rule out a role for additional protein or lipid mediators contributing to the differential effects on coagulation, particularly given our proteomic data, which found that $\mathrm{eMP}^{\mathrm{HG}}$ contain proteins associated with blood coagulation.

We also observed a significant increase in the capacity of $\mathrm{eMP}^{\mathrm{HG}}$ to stimulate ROS production and impair endotheliumdependent vasorelaxation. Similarly, Jansen et al have reported that $\mathrm{eMP}^{\mathrm{HG}}$ induce $\mathrm{ROS}$ production in cultured endothelial cells and impair vasorelaxation in $\mathrm{ApoE}^{-/-}$mice [17]. The authors found that $\mathrm{eMP}^{\mathrm{NG}}$ had no effect on ROS production or vasorelaxation. By contrast, we observed that both $\mathrm{eMP}^{\mathrm{NG}}$ and $\mathrm{eMP}^{\mathrm{HG}}$ were biologically active, with $\mathrm{eMP}^{\mathrm{HG}}$ possessing more potent biological effects. Our myography studies were conducted on healthy vessels, as opposed to $\mathrm{ApoE}^{-/-}$vessels, which establish that eMPs can exert adverse effects in the absence of underlying disease. Importantly, our studies also establish a direct, enhanced and rapid impairment of vascular function by $\mathrm{eMP}^{\mathrm{HG}}$, as treatments and assessment of reactivity were conducted ex vivo, avoiding any potential immunemediated indirect effects on vascular function.

In addition to changes in eMP formation, we observed that high glucose altered the physical composition of eMPs. $\mathrm{eMP}^{\mathrm{HG}}$ were larger than those formed under normal glucose, and the increase in size was associated with a greater negative surface charge as determined by zeta potential. The physical changes in $\mathrm{eMP}^{\mathrm{HG}}$ suggested an altered protein composition. In support of this, our proteomic analysis identified 68 proteins exclusive to $\mathrm{eMP}^{\mathrm{HG}}$. A functional assessment of $\mathrm{eMP}^{\mathrm{HG}}$ exclusive proteins by ClueGO revealed enrichment in proteins associated with haemostasis, hexose metabolism, cell surface interactions at the vascular wall and oxidation-reduction processes. The fact that proteins associated with hexose metabolism are enriched in eMPs arising from high glucose-treated cells is perhaps not surprising and suggests that eMPs are reflective of their cell of origin's ongoing response to metabolic stress. Assessment of eMP proteins may therefore represent a novel molecular signature and potential clinical biomarker indicative of subclinical glucose-induced vascular injury. We also observed an enrichment in proteins associated with haemostasis, oxidation-reduction processes and cell surface interactions at the vascular wall. We have previously reported that eMPs induce endothelial cell ROS production and expression of cell adhesion molecules [12], while Terrisse et al have reported microparticle-mediated activation of platelet interactions with the vasculature [40]. In addition, our present assessments of biological activity suggest that 
$\mathrm{eMP}^{\mathrm{HG}}$ possess augmented capacity for induction of coagulation, oxidative stress and impairments in vascular reactivity. Given the close association between many of the pathways identified in our proteomic screen and the established biological effects of microparticles, further exploration of these proteins may provide insight into the mechanisms of eMP signalling.

Our proteomic data differ slightly from related work by $\mathrm{Zu}$ et al who also conducted proteomic analysis on endothelial cellderived vesicles following high glucose treatment [41]. In their study, exposure of HUVECs to $22.5 \mathrm{mmol} / \mathrm{l}$ glucose for $4 \mathrm{~h}$ was associated with the presence of amyloid beta A4 protein and other 'Alzheimer's disease-related proteins' in their extracellular vesicle population. Of the 30 proteins listed by $\mathrm{Zu}$ et al, 11 were also identified in our screen, although none were exclusive to our high glucose population. We did not observe the presence of amyloid beta proteins in either $\mathrm{eMP}^{\mathrm{NG}}$ or $\mathrm{eMP}^{\mathrm{HG}}$, and our ClueGO did not identify Alzheimer's disease proteins as enriched. A possible explanation for the discrepancy in data between these two studies could be the differing treatment times. While $\mathrm{Zu}$ et al treated for $4 \mathrm{~h}$ prior to vesicle isolation we assessed changes after $24 \mathrm{~h}$, which may allow for more transcriptional-related changes in proteins, reflected in eMP content. Alternatively, differences in vesicle isolation methods may be responsible, as $\mathrm{Zu}$ and colleagues employed a non-selective vesicle isolation method that would not discriminate microparticles from other extracellular vesicles, such as exosomes and apoptotic bodies. It is therefore possible that enrichment in Alzheimer's disease-related proteins was related to changes in exosomes/apoptotic body protein content. Our methods included a low speed spin to remove apoptotic bodies, as well as a high speed $(20,000 \mathrm{~g})$ spin that causes microparticles to sediment but leaves exosomes in the supernatant fraction. While no centrifugation-based method will completely separate these distinct vesicle populations [4], our NTA identified $>85 \%$ of isolated vesicles as $100-1000 \mathrm{~nm}$ in size.

Our study has a number of limitations. First, our experiments were conducted in HUVEC cells. While these cells are particularly amenable to the large-scale culture necessary for our experiments, they are also fetal in origin and derive from the venous system. Thus, they may respond differently than arterial endothelial cells. Indeed, one must acknowledge that any endothelial cell population (HUVEC or otherwise) will undergo phenotypic drift when removed from its natural environment [42]. Thus, it will ultimately be important to assess the changes to eMPs in pathology in vivo. Second, these experiments were performed at a single time point. The $24 \mathrm{~h}$ exposure time was chosen to ensure sufficient time for transcriptionally dependent molecular changes in endothelial cells (and ultimately eMPs) but to avoid any induction of endothelial cell apoptosis. Nevertheless, we acknowledge that the phenotypic changes seen in $\mathrm{eMP}^{\mathrm{HG}}$ may be time-sensitive and that chronic hyperglycaemia may exert different effects.
Similarly, a relatively high concentration of glucose ( $25 \mathrm{mmol} / \mathrm{l}$ ) was used to model hyperglycaemia. This dose was chosen to ensure that endothelial cell responses over time would not be affected by glucose depletion and to maximise glucose-dependent responses in the acute setting. Ultimately, however, it will be important to confirm the high glucoseinduced changes to eMPs in the in vivo diabetic environment. Finally, L-glucose was used as an osmotic control in selected experiments; however, our proteomic data suggest that L-glucose does exert effects on endothelial cells, reflected in a change in eMP content.

The observation that eMP composition is altered depending on a cell's external microenvironment suggests that eMPs may have value as a diagnostic tool. Traditionally, increases in plasma levels of eMPs have been used as an early noninvasive biomarker of vascular injury $[7,8,43]$. Our data suggest that eMP protein content may provide insight into the molecular status of the cell from which they originate. Thus, assessment of eMP protein content could be used clinically to identify dysregulated pathways for therapeutic targeting. In addition, we observed altered bioactivity of $\mathrm{eMP}^{\mathrm{HG}}$ in the form of increased pro-coagulant and prooxidative activity. These data suggest that eMPs may actively contribute to the progression of vascular disease and that this process may be accelerated in diseases characterised by hyperglycaemia. Accordingly, inhibiting eMP-based signalling in the vasculature may represent a novel approach to the treatment of vascular complications associated with hyperglycaemia and diabetes.

In summary, we demonstrate that high glucose is a potent stimulus for eMP formation and that eMPs formed under high glucose are compositionally and functionally distinct from those formed under normal glucose conditions. Such changes may contribute to the accelerated development and progression of endothelial dysfunction in hyperglycaemia and diabetes.

Acknowledgements The authors graciously acknowledge the assistance of L. Zhu (Ottawa Hospital Research Institute) in the isolation of mesenteric vessels for wire myography. L. Puente (Ottawa Hospital Research Institute) assisted in LC-MS/MS experiments and J. Richards (Ottawa Hospital Research Institute) assisted with data formatting.

Data availability The data that support the findings of this study are available from the corresponding author upon reasonable request.

Funding This work was supported by research grants from Diabetes Canada (to DB, OG-3-14-4548-DB), the Canadian Institutes of Health Research (to DB, 362738) and the Kidney Foundation of Canada (to $\mathrm{KDB})$. DB was supported by a fellowship from the Heart and Stroke Foundation of Canada/Pfizer Canada and a Kidney Research Scientist Core Education and National Training Program (KRESCENT) New Investigator Award. 
Duality of interest The authors declare that there is no duality of interest associated with this manuscript.

Contribution statement DB and MT contributed to the study design, conducted experiments, analysed data and drafted the manuscript. FX, MNM and SA conducted experiments for the study and reviewed the manuscript. KDB contributed to the study design, analysed data and drafted the manuscript. DB is the guarantor of this work and, as such, had full access to all the data in the study and takes responsibility for the integrity of the data and the accuracy of the data analysis. All authors gave their final approval of this version of the manuscript.

\section{References}

1. Burger D, Schock S, Thompson CS, Montezano AC, Hakim AM, Touyz RM (2013) Microparticles: biomarkers and beyond. Clin Sci (Lond) 124:423-441

2. Erdbrugger U, Le TH (2016) Extracellular vesicles in renal diseases: more than novel biomarkers? J Am Soc Nephrol 27:12-26

3. Gyorgy B, Szabo TG, Pasztoi M et al (2011) Membrane vesicles, current state-of-the-art: emerging role of extracellular vesicles. Cell Mol Life Sci 68:2667-2688

4. Witwer KW, Buzas EI, Bemis LT et al (2013) Standardization of sample collection, isolation and analysis methods in extracellular vesicle research. J Extracell Vesicles 2:20360

5. Amabile N, Rautou PE, Tedgui A, Boulanger CM (2010) Microparticles: key protagonists in cardiovascular disorders. Semin Thromb Hemost 36:907-916

6. Jimenez JJ, Jy W, Mauro LM, Soderland C, Horstman LL, Ahn YS (2003) Endothelial cells release phenotypically and quantitatively distinct microparticles in activation and apoptosis. Thromb Res 109:175-180

7. Amabile N, Guerin AP, Leroyer A et al (2005) Circulating endothelial microparticles are associated with vascular dysfunction in patients with end-stage renal failure. J Am Soc Nephrol 16:33813388

8. Sinning J-M, Losch J, Walenta K, Bohm M, Nickenig G, Werner N (2011) Circulating CD31+/Annexin V+ microparticles correlate with cardiovascular outcomes. Eur Heart J 32:2034-2041

9. Loyer X, Vion AC, Tedgui A, Boulanger CM (2014) Microvesicles as cell-cell messengers in cardiovascular diseases. Circ Res 114:345-353

10. Brodsky SV, Zhang F, Nasjletti A, Goligorsky MS (2004) Endothelium-derived microparticles impair endothelial function in vitro. Am J Physiol Heart Circ Physiol 286:H1910-H1915

11. Burger D, Kwart DG, Montezano AC et al (2012) Microparticles induce cell cycle arrest through redox-sensitive processes in endothelial cells: implications in vascular senescence. J Am Heart Assoc 1:e001842

12. Burger D, Montezano AC, Nishigaki N, He Y, Carter A, Touyz RM (2011) Endothelial microparticle formation by angiotensin II is mediated via Ang II receptor type I/NADPH oxidase/Rho kinase pathways targeted to lipid rafts. Arterioscler Thromb Vasc Biol 31: 1898-1907

13. Burger D, Turner M, Munkonda MN, Touyz RM (2016) Endothelial microparticle-derived reactive oxygen species: role in endothelial signaling and vascular function. Oxidative Med Cell Longev 2016:5047954

14. Schock SC, Edrissi H, Burger D, Cadonic R, Hakim A, Thompson C (2014) Microparticles generated during chronic cerebral ischemia deliver proapoptotic signals to cultured endothelial cells. Biochem Biophys Res Commun 450:912-917
15. Peterson DB, Sander T, Kaul S et al (2008) Comparative proteomic analysis of PAI-1 and TNF-alpha-derived endothelial microparticles. Proteomics 8:2430-2446

16. Agouni A, Ducluzeau P-H, Benameur T et al (2011) Microparticles from patients with metabolic syndrome induce vascular hyporeactivity via Fas/Fas-ligand pathway in mice. PLoS One 6:e27809

17. Jansen F, Yang X, Franklin BS et al (2013) High glucose condition increases NADPH oxidase activity in endothelial microparticles that promote vascular inflammation. Cardiovasc Res 98:94-106

18. Jansen F, Wang H, Przybilla D et al (2016) Vascular endothelial microparticles-incorporated microRNAs are altered in patients with diabetes mellitus. Cardiovasc Diabetol 15:49

19. Burger D, Vinas JL, Akbari S et al (2015) Human endothelial colony-forming cells protect against acute kidney injury: role of exosomes. Am J Pathol 185:2309-2323

20. Lytvyn Y, Xiao F, Kennedy CR et al (2017) Assessment of urinary microparticles in normotensive patients with type 1 diabetes. Diabetologia 60:581-584

21. Burger D, Gagnon A, Lochnan HA, Mahzari M, Sorisky A (2015) Thyroid-stimulating hormone acutely increases levels of circulating pro-coagulant microparticles. Clin Endocrinol 83:285-287

22. Dikalov S, Griendling KK, Harrison DG (2007) Measurement of reactive oxygen species in cardiovascular studies. Hypertension 49: 717-727

23. Briones AM, Nguyen Dinh Cat A, Callera GE et al (2012) Adipocytes produce aldosterone through calcineurin-dependent signaling pathways: implications in diabetes mellitus-associated obesity and vascular dysfunction. Hypertension 59:1069-1078

24. Xiao F, Zimpelmann J, Agaybi S, Gurley SB, Puente L, Burns KD (2014) Characterization of angiotensin-converting enzyme 2 ectodomain shedding from mouse proximal tubular cells. PLoS One 9:e85958

25. Hahne H, Pachl F, Ruprecht B et al (2013) DMSO enhances electrospray response, boosting sensitivity of proteomic experiments. Nat Methods 10:989-991

26. Bindea G, Mlecnik B, Hackl H et al (2009) ClueGO: a Cytoscape plug-in to decipher functionally grouped gene ontology and pathway annotation networks. Bioinformatics 25:1091-1093

27. Shannon P, Markiel A, Ozier O et al (2003) Cytoscape: a software environment for integrated models of biomolecular interaction networks. Genome Res 13:2498-2504

28. Feng B, Chen Y, Luo Y, Chen M, Li X, Ni Y (2010) Circulating level of microparticles and their correlation with arterial elasticity and endothelium-dependent dilation in patients with type 2 diabetes mellitus. Atherosclerosis 208:264-269

29. Sabatier F, Darmon P, Hugel B et al (2002) Type 1 and type 2 diabetic patients display different patterns of cellular microparticles. Diabetes 51:2840-2845

30. Connor DE, Exner T, Ma DD, Joseph JE (2009) Detection of the procoagulant activity of microparticle-associated phosphatidylserine using XACT. Blood Coagul Fibrinolysis 20:558-564

31. Morel O, Toti F, Hugel B, Freyssinet JM (2004) Cellular microparticles: a disseminated storage pool of bioactive vascular effectors. Curr Opin Hematol 11:156-164

32. UK Prospective Diabetes Study (UKPDS) Group (1998) Intensive blood-glucose control with sulphonylureas or insulin compared with conventional treatment and risk of complications in patients with type 2 diabetes (UKPDS 33). Lancet 352:837-853

33. Lopes-Virella MF, Carter RE, Gilbert GE et al (2008) Risk factors related to inflammation and endothelial dysfunction in the DCCT/EDIC cohort and their relationship with nephropathy and macrovascular complications. Diabetes Care 31:2006-2012

34. Polak JF, Backlund J-YC, Cleary PA et al (2011) Progression of carotid artery intima-media thickness during 12 years in the diabetes control and complications trial/epidemiology of diabetes 
interventions and complications (DCCT/EDIC) study. Diabetes 60: 607-613

35. Koga H, Sugiyama S, Kugiyama K et al (2005) Elevated levels of VE-cadherin-positive endothelial microparticles in patients with type 2 diabetes mellitus and coronary artery disease. J Am Coll Cardiol 45:1622-1630

36. Cheng V, Kashyap SR, Schauer PR, Kirwan JP, McCrae KR (2013) Restoration of glycemic control in patients with type 2 diabetes mellitus after bariatric surgery is associated with reduction in microparticles. Surg Obes Relat Dis 9:207-212

37. Esposito K, Maiorino MI, Di Palo C et al (2011) Effects of pioglitazone versus metformin on circulating endothelial microparticles and progenitor cells in patients with newly diagnosed type 2 diabetes - a randomized controlled trial. Diabetes Obes Metab 13:439-445

38. Burger D, Thibodeau JF, Holterman CE, Burns KD, Touyz RM, Kennedy CR (2014) Urinary podocyte microparticles identify prealbuminuric diabetic glomerular injury. J Am Soc Nephrol 25: $1401-1407$
39. Ettelaie C, Su S, Li C, Collier ME (2008) Tissue factor-containing microparticles released from mesangial cells in response to high glucose and AGE induce tube formation in microvascular cells. Microvasc Res 76:152-160

40. Terrisse AD, Puech N, Allart S et al (2010) Internalization of microparticles by endothelial cells promotes platelet/endothelial cell interaction under flow. J Thromb Haemost 8:2810-2819

41. Zu L, Niu C, Li J, et al. (2015) Proteomic research of high-glucoseactivated endothelial microparticles and related proteins to Alzheimer's disease. Diab Vasc Dis Res 12:467-470

42. Aird WC (2007) Phenotypic heterogeneity of the endothelium: I. Structure, function, and mechanisms. Circ Res 100:158-173

43. Amabile N, Guerin AP, Tedgui A, Boulanger CM, London GM (2012) Predictive value of circulating endothelial microparticles for cardiovascular mortality in end-stage renal failure: a pilot study. Nephrol Dial Transplant 27:1873-1880 